\title{
Deriving the Dependence Structure of Portfolio Credit Derivatives Using Evolutionary Algorithms
}

\author{
Svenja Hager and Rainer Schöbel \\ Department of Corporate Finance, \\ Faculty of Economics and Business Administration, \\ Eberhard-Karls-University of Tübingen, \\ Mohlstraße 36, 72074 Tübingen, Germany
}

\begin{abstract}
The correct modeling of default dependence is essential for the valuation of portfolio credit derivatives. However, for the pricing of synthetic CDOs a one-factor Gaussian copula model with constant and equal pairwise correlations for all assets in the reference portfolio has become the standard market model. If this model were a reflection of market opinion there wouldn't be the implied correlation smile that is observed in the market. The purpose of this paper is to derive a correlation structure from observed CDO tranche spreads. The correlation structure is chosen such that all tranche spreads of the traded CDO can be reproduced. This implied correlation structure can then be used to price off-market tranches with the same underlying as the traded CDO. Using this approach we can significantly reduce the risk to misprice offmarket derivatives. Due to the complexity of the optimization problem we apply Evolutionary Algorithms.
\end{abstract}

\section{Introduction}

Although it is still of interest to find empirical sources of correlation data, people increasingly use the market of synthetic collateralized debt obligations (CDOs) to derive information about the correlation structure of the underlying of a CDO. An observed CDO premium can be interpreted as an indicator of asset correlation. Therefore, more and more tranched products are quoted in terms of an implied correlation parameter instead of the spread or the price of a tranche. The implied correlation of a tranche is the uniform asset correlation that makes the tranche spread computed by the standard market model equal to its observed market spread. The standard market model is a Gaussian copula model that uses only one single parameter to summarize all correlations among the various borrowers' default times. But obviously a flat correlation structure is not able to reflect the heterogeneity of the underlying asset correlations since the complex relationship between the default times of different assets can't be expressed in one single number. Obviously, the standard market model doesn't reflect market opinion because the implied correlation smile emerges. Typically, mezzanine 
tranches trade at lower implied correlations than equity and senior tranches on the same portfolio. This phenomenon is called implied correlation smile. Despite the questionable explanatory power of the implied correlation parameter, the implied correlation of a CDO tranche is often used to price off-market products with the same underlying as the traded CDO.

Recently, more and more researchers examine different approaches to explain and model the correlation smile. Gregory and Laurent 2] and Andersen and Sidenius [1] introduce dependence between recovery rates and defaults. In a second extension Andersen and Sidenius introduce random factor loadings to permit higher correlation in economic depressions. Both approaches are able to model a smile. Hull and White [4] discuss the effect of uncertain recoveries on the specification of the smile.

The correlation smile shows clearly that it is not appropriate to use the implied correlation of a traded CDO tranche to value non-standard tranches on the same collateral pool. To address these shortcomings we take the implied correlation approach one step further and imply a correlation matrix that reproduces all given tranche spreads of a CDO simultaneously. The dependence structure is chosen such that the resulting tranche prices are concordant with observed market prices or, respectively, such that the observed correlation smile is reproduced. Hager and Schöbel [3] showed that heterogeneous correlation structures are able to model a smile. After we derived a suitable asset correlation structure, we can use this dependency to price off-market products with the same underlying. In this study we use Evolutionary Algorithms (EAs) to derive a dependence structure from observed CDO tranche spreads. We show systematically why EAs are suitable for this kind of application. So far, there is only a limited amount of studies that connect EAs with derivative pricing. To our knowledge we are the first to apply EAs to the implied correlation problem and we are the first to derive a correlation matrix that is not necessarily flat from a set of observed tranche spreads.

\section{The Optimization Problem}

Suppose for the moment that we know the tranche spreads of an actively traded CDO. We assume that the CDO consists of an equity tranche, a mezzanine tranche and a senior tranche. Our goal is to derive a correlation matrix $\Sigma$ that replicates the given tranche spreads of the equity, the mezzanine and the senior tranche simultaneously. Denote these target values as $\overline{s_{e}}, \overline{s_{m}}$ and $\overline{s_{s}}$. It is intuitively clear that in general there can be more than one correlation matrix that leads to the respective tranche spreads $\overline{s_{e}}, \overline{s_{m}}$ and $\overline{s_{s}}$. Hager and Schöbel [3] discuss this subject. Note that there might also be combinations of tranche spreads that can't be reproduced by any correlation matrix. However, there is no way to derive the correlation matrix $\Sigma$ in closed form since even the portfolio loss distribution can't be computed in closed form for arbitrary correlation matrices.

To measure the quality of an obtained correlation matrix $\Sigma$, we first compute the appendant equity, mezzanine and senior tranche spreads $s_{e}(\Sigma), s_{m}(\Sigma)$ 
and $s_{s}(\Sigma)$ and compare them with the given target values $\overline{s_{e}}, \overline{s_{m}}$ and $\overline{s_{s}}$. The goal is to find a correlation matrix such that the corresponding spreads agree. The optimization problem discussed in this study is rather complex because the search space is high dimensional and multi-modal and the objective function is non-linear, non-differentiable and discontinuous. Note that for arbitrary correlation matrices often both $s_{e}(\Sigma), s_{m}(\Sigma), s_{s}(\Sigma)$ and $\overline{s_{e}}, \overline{s_{m}}, \overline{s_{s}}$ are obtained via Monte-Carlo simulation. In this case we have to deal with noise. Since our optimization problem is characterized by these properties the number of applicable optimization techniques is restricted. Therefore, we choose EAs to address this challenging problem. EAs are stochastic search methods that are inspired by the Darwinian theory. They model the collective learning process within a population. The starting population is generally initialized by random. In the course of the generations the population is supposed to evolve toward successively better regions of the search space by randomized processes of selection, recombination and mutation. The generations are searched until a sufficiently good solution is found or until a termination criterion is met. Similar to other heuristic search methods, it is not guaranteed that EAs find the global optimum, but they generally find good solutions in a reasonable amount of time.

Consider the function $f(\Sigma)$ which measures the sum of the relative deviations of the obtained tranche spreads from the target spreads:

$$
f(\Sigma)=\frac{\left|s_{e}(\Sigma)-\overline{s_{e}}\right|}{\overline{s_{e}}}+\frac{\left|s_{m}(\Sigma)-\overline{s_{m}}\right|}{\overline{s_{m}}}+\frac{\left|s_{s}(\Sigma)-\overline{s_{s}}\right|}{\overline{s_{s}}} .
$$

In our optimization problem low values of $f(\Sigma)$ stand for high quality. In a population based optimization strategy with $\lambda$ individuals, we neglect the overall performance of a certain generation and just consider the best individual in the respective generation. The objective function registers the quality of the best individual that has been generated so far. Let $h(t)$ denote the objective function at time $t$ and let $\Sigma^{k, \tau}$ denote the $k^{t h}$ individual in generation $\tau, k \in\{1, \ldots, \lambda\}$, $\tau \in\{1, \ldots, t\}$. Consequently, the objective function, that has to be minimized, is

$$
h(t)=\min _{k \in\{1, \ldots, \lambda\}, \tau \in\{1, \ldots, t\}}\left(f\left(\Sigma^{k, \tau}\right)\right) .
$$

\section{Pricing of Synthetic CDOs}

In this study we always assume that the intensity based approach describes the default of one obligor and that the Gaussian copula model with an arbitrary correlation matrix describes the dependency between the obligors' default times. In our optimization problem all model parameters are known except for the pairwise linear correlations.

Following Laurent and Gregory [5] we consider a synthetic CDO, whose underlying consists of $n$ reference assets, and assume that asset $j$ has exposure $\frac{1}{n}$ and default time $\tau_{j} . N_{j}(t)=1_{\left\{\tau_{j} \leq t\right\}}$ denotes the default indicator process. The cumulative portfolio loss at time $t$ is therefore $L(t)=\frac{1}{n} \sum_{j=1}^{n} N_{j}(t)$. A CDO is 
a structured product that can be divided into various tranches. The cumulative default of tranche $(A, B), 0 \leq A<B \leq 1$ is the non-decreasing function

$$
\omega(L(t))=(L(t)-A) 1_{[A, B]}(L(t))+(B-A) 1_{] B, 1]}(L(t)) .
$$

The tranche spread $s_{(A, B)}$ depends on the thresholds $A$ and $B$. Let $B(t)$ be the discount factor for maturity $t$. $T$ stands for the maturity of the CDO and let $t_{1}, \ldots, t_{I}$ denote the regular payment dates for the CDO margins.

The default payments can be written as

$$
E\left[\sum_{j=1}^{n} B\left(\tau_{j}\right) N_{j}(T)\left(\omega\left(L\left(\tau_{j}\right)\right)-\omega\left(L\left(\tau_{j}^{-}\right)\right)\right)\right] .
$$

The margin payments are based on the outstanding nominal of the tranche. Since defaults often take place between regular payment dates we have to distinguish between the regular payments

$$
s_{(A, B)} \sum_{i=1}^{I} B\left(t_{i}\right) E\left[B-A-\omega\left(L\left(t_{i}\right)\right)\right]
$$

and the accrued payments. Accrued margins are paid for the time from the last regular payment date before $\tau_{j}$ (this date is called $t_{k(j)-1}$ ) until $\tau_{j}$. The accrued payments can be written as

$$
s_{(A, B)} E\left[\sum_{j=1}^{n} B\left(\tau_{j}\right) N_{j}(T)\left(\tau_{j}-t_{k(j)-1}\right)\left(\omega\left(L\left(\tau_{j}\right)\right)-\omega\left(L\left(\tau_{j}^{-}\right)\right)\right)\right] .
$$

A synthetic CDO can be compared with a default swap transaction because the CDO margin payments are exchanged with the default payments on the tranche. The spread $s_{(A, B)}$ is derived by setting the margin payments in (2) and (3) and the default payments in (1) equal.

\section{Experimental Settings and Results}

\subsection{Description of the Genotype}

Correlation matrices are symmetric positive semi-definite matrices whose matrix elements are in $[-1,1]$. The diagonal of a correlation matrix always consists of ones. In the following, $\Sigma=\left(\Sigma_{i j}\right)_{i, j=1, \ldots, n}$ denotes the correlation matrix. We use $\Sigma$ as phenotype and a row vector $\rho=\left(\rho_{i}\right)_{i=1, \ldots, n} \in[-1,1]^{n}$ as realvalued genotype. The pairwise linear correlation between asset $i$ and asset $j$ can be computed as $\Sigma_{i j}=\rho_{i} \rho_{j}, i \neq j$ and $\Sigma_{i j}=1, i=j$. Using this socalled one-factor approach we can avoid Monte-Carlo simulations and provide semi-explicit expressions for CDO tranche spreads (see Laurent and Gregory [5]). Note that there are correlation matrices that can't be represented by the one-factor approach. 
The initial population consists of randomly generated vectors $\rho \in[-1,1]^{n}$. An arbitrary vector $\rho$ automatically leads to a symmetric, positive semi-definite matrix with elements $\Sigma_{i j}=\rho_{i} \rho_{j}, i \neq j$ and $\Sigma_{i j}=1, i=j$.

In this study we compare several standard recombination and mutation schemes. They are carried out according to custom.

Note that recombination and mutation can breed vector elements with $\left|\rho_{i}\right|>$ 1. To make sure that the pairwise correlations are in $[-1,1]$, define a censored vector $\rho^{*}=\left(\rho_{i}^{*}\right)_{i=1, \ldots, n}$ with $\rho_{i}^{*}=\min \left(\max \left(\rho_{i},-1\right), 1\right)$ that replaces $\rho$. We maintain this modified representation of the genotype.

We consider two cases of suitable termination conditions. Naturally, reaching the optimum of the objective function with a certain precision should be used as stopping condition. Therefore, we stop our algorithm as soon as the objective function falls below a predefined value. Furthermore, we terminate the EA when the total number of function evaluations reaches a given limit.

\subsection{Setup}

To assess the potential of our approach, we work with simulated data. We compare the performance of a Monte-Carlo Search, a Hill-Climber $((1+1)$-ES), an Evolution Strategy with 4 parent and 20 offspring individuals $((4,20)$-ES) and a generational Genetic Algorithm with 40 individuals (GA(40)). We mutate individuals by adding realizations of normally distributed random variables with expected value 0 and standard deviation 0.05 unless explicitly mentioned otherwise. We apply global mutation operators, i.e. every vector element is subject to mutation. In case of the $(4,20)$-ES we use elite selection and 1-point crossover. Our focus is on the application of different mutation operators. We consider the 1/5-rule, global mutation without a strategy parameter and global mutation with a strategy parameter that controls the mutation step size. The mutation probability is 0.95 , the crossover probability is 0.50 . In case of the GA(40) we focus on the selection and crossover parameters. We use proportional selection and tournament selection with a tournament group size of 10 . We use 1-point crossover and intermediate crossover and we use global mutation without a strategy parameter. The crossover probability is 0.95 , the mutation probability is 0.50 . In our study we just consider non-negative pairwise linear correlations and therefore non-negative genotypes for the sake of simplicity. As soon as the objective function falls below $5 \%$ we terminate the algorithm. At most 2000 function evaluations are carried out.

We consider a CDO that consists of three tranches. The respective attachment and detachment levels are 0\%-5\%,5\%-15\%, 15\%-100\%. We assume that the underlying is a homogeneous pool of 10 names with equal unit nominal. The default intensity of each obligor is $1 \%$, the recovery rate is $40 \%$. The time to maturity is 5 years. Our goal is to find a correlation matrix that models an observed compound correlation smile. The given implied compound correlations are 0.24 for the equity tranche, 0.05 for the mezzanine tranche and 0.35 for the senior tranche. At first, we compute the spreads of the equity, the mezzanine and the senior tranche using the respective implied correlations. We get 802.2 
bps, 204.4 bps and 9.0 bps. Then, we have to find a correlation matrix that reproduces all three tranche spreads simultaneously.

\subsection{Performance}

To make sure that we obtain reliable results, we repeat each implementation 25 times. We compute the mean value of the objective functions over the 25 runs, and we also consider the $10 \%$ and the $90 \%$ quantiles. Our focus is on the decline of the objective function in the course of the generations. To compare the performance of the different algorithms consider figure 1. It shows the objective functions for the different implementations.

At first we compare a Monte-Carlo search and a $(1+1)$-ES to analyze the search space. Generally, the Monte-Carlo search is rather inefficient especially in high dimensional search spaces. Whenever the Monte-Carlo search performs as well as a Hill-Climber or a population-based EA, the search space is probably very flat or very cragged or non-causal. However, in our case the Hill-Climbing strategy clearly outperforms the random search. Often, $(1+1)$-strategies are very efficient in simple unimodal search spaces. But if a Hill-Climber starts up the wrong hill, it has no chance to know that it has found an inferior optimal solution. Therefore, Hill-Climbing strategies frequently can't handle situations in which there are several local optima.

Then we extend the $(1+1)$-ES to a multistart $(1+1)$-ES. We obtain several different solution matrices (see figure 2 for two examples). These matrices yield tranche spreads that are sufficiently close to $802.2 \mathrm{bps}, 204.4 \mathrm{bps}$ and $9.0 \mathrm{bps}$, i.e. the sum of the percentual deviations is less than $5 \%$. This leads to the conclusion that the search space is multimodal. A multistart $(1+1)$-ES reduces the risk of premature convergence.

We now compare different implementations of a $(4,20)$-ES and a GA(40). The performance of the different ES implementations is nearly identic, the confidence intervals widely overlap. There is only a very small difference, but the global mutation strategy with one strategy parameter outperforms the other approaches. Then we compare the different GA implementations. The performance of the different GA implementations differs considerably. The GA with tournament selection combined with 1-point crossover leads to the best result. The GA with proportional selection and 1-point crossover performs moderately. The GA with proportional selection and intermediate crossover converges too fast to a dissatisfactory solution. This implementation yields the worst result. It is important to know that by means of recombination the hyperbody formed by the parents generally can't be left by the offspring individuals. Especially the intermediate crossover technique causes volume reduction since it successively narrows the search space, such that eventually the optimal solution can't be attained any more after a few generations.

We finally compare all algorithms on the basis of function evaluations needed to obtain a sufficiently good result. The $(1+1)$-ES performs slightly better than the $(4,20)$-ES implementations, there is only a very small difference. However, the GA implementations cant keep up with the ES implementations. 


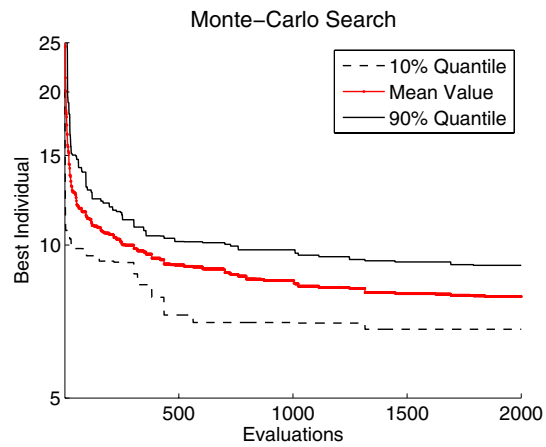

$(4,20)-E S$, Global Mutation, 1/5-Rule

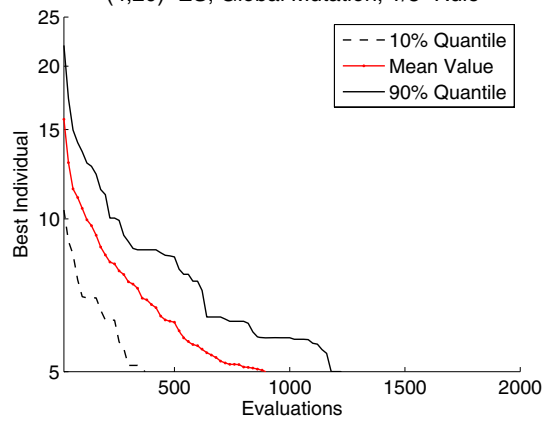

$(4,20)-E S$, Global Mutation Without Strategy Param.

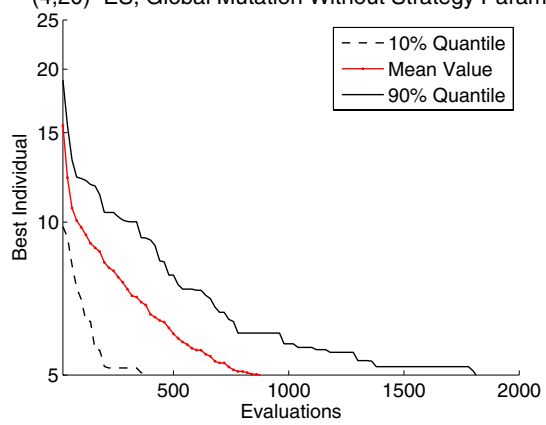

GA(40), Proportional Selection, Interm. Crossover

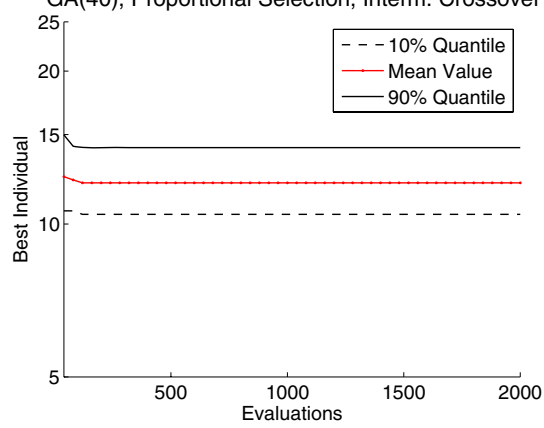

(1+1)-ES

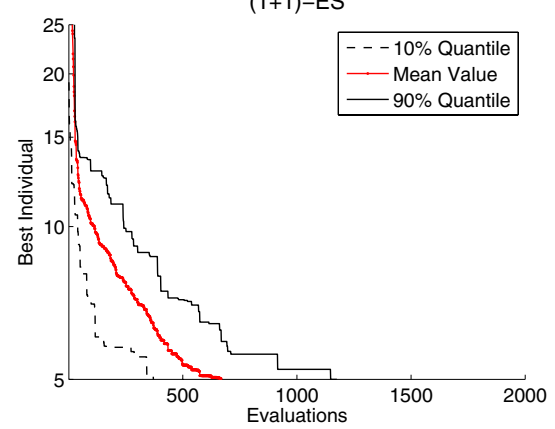

$(4,20)-E S$, Global Mutation With Strategy Parameter

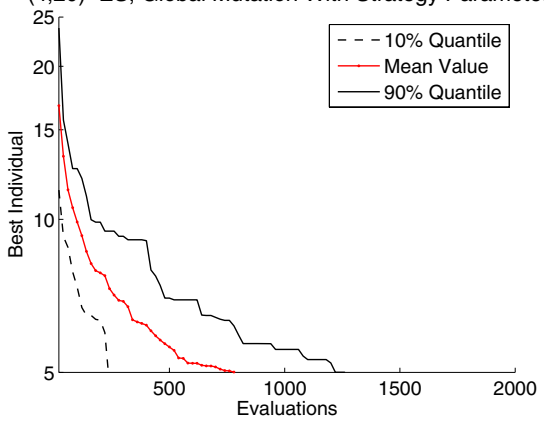

GA(40), Proportional Selection, 1-Point Crossover

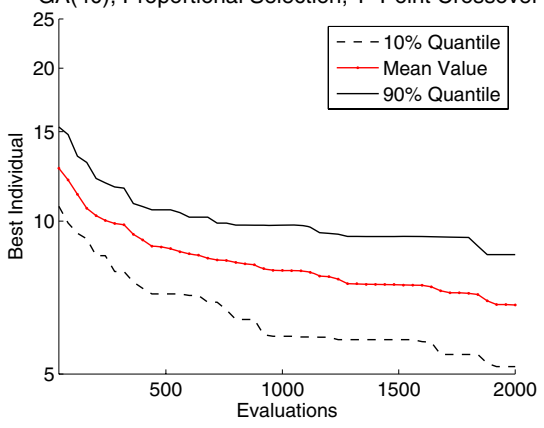

GA(40), Tournament Selection, 1-Point Crossover

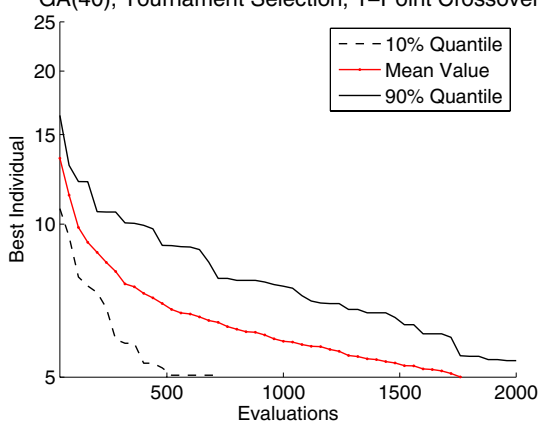

Fig. 1. Performance of different algorithms 

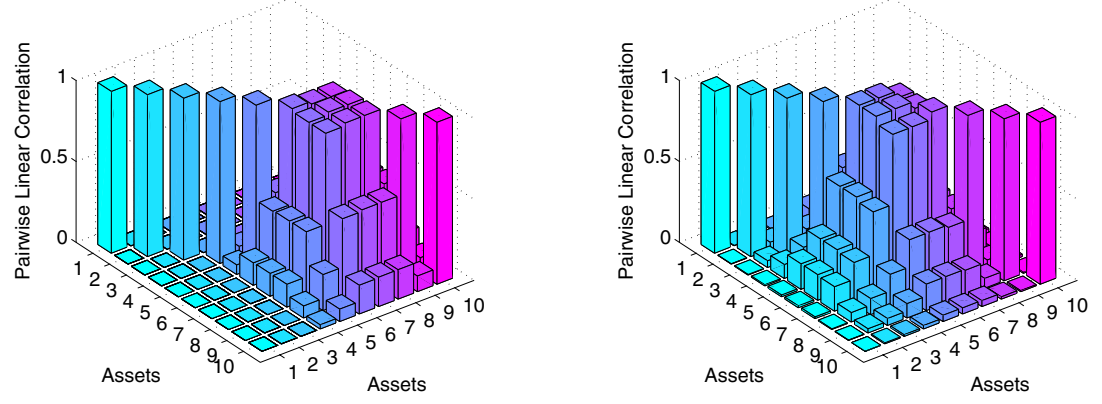

Fig. 2. Exemplary correlation matrices

\section{Conclusion}

In this study we used the concept of Evolutionary Algorithms to derive possible dependence structures of the underlying of a traded CDO from observed tranche spreads. These dependence structures can then be used to price off-market products with the same underlying as the CDO. Using this strategy we can reduce the pricing discrepancy that comes up when the implied correlation approach is applied. We presented several implementations of Evolutionary Algorithms and discussed their performance.

\section{References}

1. Andersen, L., Sidenius, J.: Extensions to the Gaussian copula: Random recovery and random factor loadings. The Journal of Credit Risk 1(1) (2004) 29-70

2. Gregory, J., Laurent, J.P.: In the core of correlation. Risk, October (2004) 87-91

3. Hager, S., Schöbel R.: A note on the correlation smile. Tübinger Diskussionsbeitrag 297 (2005)

4. Hull, J., White, A.: Valuation of a CDO and an $n^{\text {th }}$ to default CDS without MonteCarlo simulation. The Journal of Derivatives 12(2) (2004) 8-23

5. Laurent, J.P., Gregory, J.: Basket default swaps, CDOs and factor copulas. The Journal of Risk 7(4) (2005) 103-122 